Revue québécoise de linguistique

--> Voir l'erratum concernant cet article

\title{
Quelques remarques sur un parler français méconnu de l'Ouest canadien : le métis
}

\section{Robert A. Papen}

Volume 14, numéro 1, 1984

Grammaires en contact

URI : https://id.erudit.org/iderudit/602530ar

DOI : https://doi.org/10.7202/602530ar

Aller au sommaire du numéro

Éditeur(s)

Université du Québec à Montréal

ISSN

0710-0167 (imprimé)

1705-4591 (numérique)

Découvrir la revue

Citer cet article

Papen, R. A. (1984). Quelques remarques sur un parler français méconnu de l'Ouest canadien : le métis. Revue québécoise de linguistique, 14(1), 113-139.

https://doi.org/10.7202/602530ar d'utilisation que vous pouvez consulter en ligne. 


\title{
QUELQUES REMARQUES SUR UN PARLER FRANÇAIS MÉCONNU DE L'OUEST CANADIEN : LE MÉTIS
}

\author{
Robert A. Papen
}

\section{Introduction}

Si les divers parlers vernaculaires français de l'Amérique du Nord ont fait, et font encore, l'objet d'études de plus en plus nombreuses, il en existe un qui a toujours échappé à l'attention de la plupart des linguistes et qui demeure donc largement inconnu. Nous faisons référence au métis, ou plus précisément au métif (prononcé [mIt $\left.\int \mathrm{If}\right]$ ), comme le désignent les locuteurs de ce parler.

Le métis se parle surtout dans les trois provinces de l'Ouest canadien, dites 'les provinces des Prairies'. Il existe également quelques locuteurs du métis dans les Territoires du Nord-Ouest et dans les États américains du Dakota du Nord et du Montana.

Nous ne tracerons pas ici l'histoire fascinante de la «nation métis» des Bois-brûlés, comme ils se désignaient autrefois - tant au Manitoba qu'en Saskatchewan ou en Alberta. Il suffit de dire que les Métis francophones ${ }^{1}$ habitent encore au Manitoba, berceau de la nation métisse,

1. Il est difficile d'établir de façon certaine l'origine généalogique des Métis «francophones». Vraisemblablement, les premiers enfants métis virent le jour durant la première moitié du XVIII ${ }^{\mathrm{e}}$ siècle puisque c'est durant cette période (1730-1745) que l'homme blanc (les frères de la Vérendrye et leurs compagnons) pénétra pour la première fois dans l'Ouest canadien. Les récits de ces voyages laissent clairement comprendre que nombreux de ces hommes ne revinrent pas au Bas-Canada et avaient préféré s'établir dans l'Ouest où, il ne faut pas en douter, ils formèrent des alliances avec des femmes autochtones de tribus diverses selon la région (cris, ojibwa, chippewa, assiniboine). Plus tard, lorsque la traite des fourrures fut bien établie par les compagnies de la Baie d'Hudson et du Nord-Ouest, la plupart des trappeurs et des guides de celle-ci étaient des Métis francophones. La compagnie de la Baie d'Hudson, pour sa part, embauchait surtout des Écossais et des Irlandais. Toutefois, selon de Trébaudan (1936), ceux- 
le long des grands lacs manitobains et aussi à Saint-Laurent, à SaintEustache, à Sainte-Anne-des-Chênes et à Saint-Lazarre. En Saskatchewan, ils sont établis surtout dans la région de Batoche, Saint-Laurent, Duck Lake et Saint-Louis, bien qu'au XIX $\mathrm{X}^{\mathrm{e}}$ siècle on les retrouve également plus au sud, dans la région de Qu'Appelle. En Alberta, c'est au Lac-la-Biche qu'on peut encore en rencontrer, ceux de l'ancien Saint-Paul-des-Métis étant pour la plupart soit disparus, soit complètement assimilés à la population franco ou anglo-albertaine.

Les localités métisses du Manitoba datent d'au moins 1809, année de la fondation du Fort Gibraltar sur la rivière Rouge alors que celles de la Saskatchewan et de l'Alberta datent de la seconde moitié du XIX $\mathrm{X}^{\mathrm{e}}$ siècle$^{2}$. Par contre, comme la plupart des isolats francophones nord-américains, le parler métis est présentement en voie de disparition dans de nombreuses localités où il se parlait couramment autrefois et il ne semble résister à l'assimilation, anglophone surtout, que dans les quelques petites localités manitobaines identifiées ci-dessus.

\section{Le métis face aux autres parlers français d'Amérique}

Dans sa classification des parlers français en Amérique du Nord, Haden (1973) fait la répartition suivante : les dialectes A de la province du Québec (le québécois), les dialectes B des provinces maritimes, des îles de la Madeleine et de l'archipel Saint-Pierre-et-Miquelon (l'acadien), le dialecte C de la Louisiane - à ne pas confondre avec le parler dit 'cajun', lui-même issu du dialecte $B$ - et le dialecte $D$, parlé dans une seule petite localité de l'État de Pennsylvanie. Le dialecte $\mathrm{C}$ était le français employé jusqu'au début du siècle par les colons d'origine française établis à la NouvelleOrléans, les soi-disant 'Créoles'. Ce parler est maintenant disparu. Le dialecte D se parle encore dans le village de Frenchville, en Pennsylvanie, fondé en 1830 par des bûcherons et des fermiers français venus surtout des

ci avaient l'habitude de quitter le pays une fois leur travail terminé, abandonnant leurs femmes indiennes et leurs enfants que des Métis français adoptaient souvent tout en les francisant. Ceci expliquerait pourquoi tant de familles métisses de langue française portent des noms d'origine écossaise ou irlandaise.

2. Selon Douaud (1980), les missionnaires Oblats avaient établi une mission au Lac-la-Biche avec une école dirigée par des sœeurs, des frères et des prêtres français, belges et québécois, afin de desservir leurs ouailles métis dès le début de 1850, ce qui indique que les Métis y étaient déjà bien établis à cette époque. 
départements de la Haute-Marne, de la Haute-Saône, des Vosges et du Haut-Rhin ${ }^{3}$.

Haden classe tous les autres parlers français du continent sous la rubrique 'diaspora', soit du québécois, soit de l'acadien. Selon lui, les francophones du Québec auraient émigré vers l'Ouest (l'Ontario, les provinces de l'Ouest et la Colombie britannique), vers le sud-ouest (DetroitWindsor, l'Illinois et le Missouri), vers le sud-est (le Nouveau-Brunswick) et vers le sud (les États de la Nouvelle-Angleterre). De l'Acadie originale, la Nouvelle-Écosse actuelle, les locuteurs du dialecte B se sont dispersés dans les autres provinces maritimes, surtout le Nouveau-Brunswick, en NouvelleAngleterre, où ils ont été assimilés par les locuteurs du parler québécois, au Québec, surtout en Gaspésie, aux îles de la Madeleine et à Montréal et en Louisiane, où le dialecte a évolué pour devenir les parlers qu'on regroupe aujourd'hui sous le vocable 'cajun'4.

Quant aux parlers québécois de la diaspora parlés en Ontario et dans les provinces de l'Ouest, Haden ne se trompe pas lorsqu'il constate que partout, on parle à peu près le même dialecte. S'il existe certaines particularités dans une région ou dans un village donné, celles-ci sont relativement marginales et si certains traits semblent caractériser globalement les 'parlers de l'Ouest', ceux-ci sont dus surtout à des phénomènes de transfert linguistique : anglicismes, calques, etc. ${ }^{5}$ Il n'en reste pas moins que ces

\section{Valdam (1974).}

4. La seule localité du Missouri où le français se parle encore est le village de Old Mines (Vieilles Mines) dans le comté de Sainte-Geneviève au sud de la ville de Saint-Louis. Haden n'inclut dans sa classification ni les parlers français des Antilles ni les créoles à base lexicale française. Parmi les premiers il faudrait mentionner le dialecte parlé à Northside, sur l'île de Saint-Thomas aux îles Vierges ainsi que celui du quartier de Frenchtown à Charlotte Amalie, capitale de Saint-Thomas. Il faudrait aussi inclure les parlers français (et non créoles) d'Haïti et des autres îles antillaises. Finalement, Haden passe sous silence les communautés québécoises installées depuis quelques années en Floride et dans les États du Sud-Ouest américain, particulièrement la Californie et l'Arizona.

5. Ainsi, même si certains items lexicaux se retrouvent tant au Québec que dans l'Ouest, le référent n'est pas toujours identique. Au Québec, le terme élévateur (à grains) fait référence à d'énormes silos en ciment qu'on retrouve surtout dans les ports et qui servent à emmagasiner le grain. Dans l'Ouest, ce terme fait référence à des silos à grains construits en bois qu'on retrouve dans tous les villages le long de la voie ferrée et dont la forme est tout à fait unique. Ceci est également vrai pour le terme cabousse (caboose 〈cab house?) qui en québécois désigne uniquement le wagon-frein alors que dans l'Ouest, outre cette acceptation, il fait référence à une petite cabane installée sur traîneaux tirée par des chevaux et servant de véhicule durant l'hiver. 
parlers sont fondamentalement identiques au(x) parler(s) québécois. D'où l'importance du métis puisque ce parler se distingue suffisamment des autres que tout locuteur francophone, si peu versé en matière dialectologique soit-il, n'aura aucune difficulté à le reconnaître comme étant effectivement «différent».

Valdam (1974) divise les communautés francophones des Amériques en cinq groupes selon un point de vue d'écologie linguistique plus que de parenté dialectale. Le Groupe 1 inclut les parlers créoles en contact avec le français (Haïti et les D.O.M. antillais); le Groupe 2 inclut les créoles en contact avec l'anglais (la Dominique, Sainte-Lucie); le Groupe 3 comprend les parlers 'laurentiens' (le québécois) et acadiens du Canada et le Groupe 4 regroupe les parlers de la Nouvelle-Angleterre et de la Louisiane. Le Groupe 5 comprend tous les isolats linguistiques «évoluant dans un environnement linguistique anglophone et dont les liens avec les autres communautés francophones sont demeurés coupés ${ }^{6} . "$ Parmi ceux-ci, Valdman inclut les parlers de Saint-Thomas, îles Vierges, celui de Vieilles-Mines au Missouri et celui de Frenchville en Pennsylvanie. Il ne fait aucune mention des parlers français de l'Ouest canadien mais on peut supposer qu'il les classerait dans le Groupe 4 puisque ceux-ci sont caractérisés par le fait qu'ils «évoluent face à des institutions qui privilégient l'anglais mais qui ont toutefois conservé un certain contact avec le français standard et d'autres parlers vernaculaires français par un apport humain ininterrompu et par des relations culturelles continues ${ }^{7} »$.

Par contre, le métis pose un problème pour la classification de Valdman. On ne peut le considérer comme un isolat total (Groupe 5) puisque les Métis ont toujours maintenu un certain contact avec les autres communautés francophones de l'Ouest. Mais le métis se classe tout aussi difficilement dans le Groupe 4 puisque, bien qu'il ait évolué dans un milieu institutionnel privilégiant l'anglais tout en maintenant un contact avec d'autres parlers français, il a également évolué en maintenant un contact avec diverses langues amérindiennes qui l'ont d'ailleurs profondément influencé, comme nous nous proposons de montrer.

Le métis offre donc au linguiste un intérêt particulier. D'une part, ce parler se distingue considérablement des autres parlers français du continent au niveau phonologique, au niveau morpho-syntaxique et au niveau lexico-

6. Valdman 1974, p. 44

7. Valdman 1974, p. 44 
sémantique. Ces différences sont le plus souvent dues au contact des diverses langues amérindiennes qui l'ont fortement marqué, surtout le cris. D'autre part, le métis conserve de nombreux traits linguistiques qui l'apparentent de toute évidence au parler québécois, même si ceux-ci semblent surtout refléter un état plus ancien de la «langue mère».

\section{Le métis et le michif}

Si le métis a été marqué par des langues amérindiennes, il ne faut pas en déduire que c'est une langue mixte. Il existe d'ailleurs un autre parler, appelé michif, qui est en fait une véritable langue mixte, un pidgin. Ce parler existe encore dans la réserve indienne de Turtle Mountain au Dakota du Nord et selon nos informations, de nombreux Métis francophones du Manitoba le parlent couramment. Selon Crawford $(1979,1983 a, b)$ le syntagme nominal du michif serait surtout du domaine phonologique, morphologique et lexical du français alors que le syntagme verbal serait du domaine du cris, avec toutes les complexités flexionnelles qu'on lui connaît, bien que certains locuteurs semblent simplifier le système verbal en ayant recours à des constructions prépositionnelles basées sur le français. À noter aussi que si le substantif est marqué pour le genre ([ \pm masculin]) par le choix approprié du déterminant (un/une ou le/la), le verbe michif maintient, comme en cris, un système d'affixes marquant l'accord avec le genre cris ([ \pm animé]) du sujet. Le substantif est donc marqué formellement selon qu'il est masculin ou féminin par le choix du déterminant et selon qu'il est animé ou inanimé par le choix approprié de l'affixe verbal. Le pronom démonstratif sera marqué pour les deux types de genre.

Le seul exemple que nous possédons est le suivant ${ }^{8}$ :

Ain om keeyayow, see zhvoo ewanihawt. Keeshiway - etayw,

[z̃nomkijajow sizvo ewanl hat ki]lwe etew]

UN HOMME IL-Y-AVAIT, SES CHEVAUX IL-AVAIT PERDU. IL-SORTIT

doudounawayw mawka la brem keeyayow.

[dUdUnıwew mak $\Lambda$ la brem kij $\Lambda$ jow]

IL-ALLA-ÇA-ET-LÀ MAIS LA BRUME IL-Y-AVAIT.

8. Exemple cité par Crawford (1979). L'orthographe employée a été développée par les locuteurs du michif de la réserve Turtle Mountain. Nous renvoyons le lecteur au dictionnaire du michif, édité par Crawford en 1983, que nous n'avons malheureusement pu consulter nousmême. 


\section{Les données}

Les quelques commentaires qui suivent ne s'adressent donc pas à ce parler michif, qui ne peut de toute évidence être considéré comme étant un parler français. Nous voulons également souligner le fait que nous n'offrons pas ici une analyse complète et détaillée du parler métis, les données disponibles étant nettement insuffisantes. Ainsi, la plupart, sinon la totalité, des traits caractéristiques du métis sont sujets à une assez grande variabilité dont il ne faudrait d'ailleurs pas s'étonner. Le corpus dont nous disposons ne nous permet donc qu'une analyse préliminaire.

Les données sur lesquelles se basent nos remarques ont été recueillies auprès d'une demi-douzaine de locuteurs habitant la région de Batoche, en Saskatchewan. Tous ces locuteurs étaient relativement âgés, le plus vieux ayant 97 ans et le plus jeune, 80 ans. Des quatre locuteurs principaux, seul l'aîné déclarait pouvoir encore parler cris, les autres étant soit monolingues français, soit bilingues français-anglais ${ }^{9}$.

\section{Le système phonologique du métis}

5.1 Le système des voyelles du métis présente un certain nombre de détails qui le relient aux autres parlers français du continent alors que d'autres le démarquent nettement. Ainsi, en métis, les voyelles fermées ont une variante relâchée en syllabe fermée, tout comme un québécois $([\mathrm{I}],[\mathrm{Y}]$, et $\mathrm{U})$. On peut même entendre la voyelle relâchée en syllabe ouverte ${ }^{10}$. Par contre, le métis ne connaît pas les diphtongues typiques du québécois.

Le phénomène le plus marquant du métis est la fermeture plus ou moins systématique des voyelles moyennes, surtout en syllabe ouverte. Nous avons noté [1I] le, les, [mI] mes, [sI] ce, ces, ses, c'est, [bll] blé, [grU] gros, [flkU] chicot, [kUtfI] côté, [kIUt]lr] clôture, [pUvr] pauvre et, bien entendu [mlt]If] métis ${ }^{11}, 12$.

9. Bien que nous n'ayons pu le vérifier de façon certaine, il est plus que probable que notre locuteur âgé ait fait référence au michif et non au cris puisqu'il admettait avoir des difficultés à comprendre ce qu'il appelait le cris "pur».

10. Selon Douaud (1980), le métis n'aurait que des réalisation en [I] et [U] pour les voyelles fermées. Nos informateurs variaient considérablement entre [i] et $[\mathrm{I}]$ et $[\mathrm{u}]$ et $[\mathrm{U}]$, surtout en syllable ouverte finale.

11. On remarquera que l'allophone [I] de /e/ déclenche l'affrication palatale d'une dentale précédente, tout comme le fait $/ \mathrm{i} /$. Voir 5.2.

12. Selon certains textes (Préfontaine 1980), le phénomène contraire - l'ouverture des voyelles fermées $(/ \mathrm{i} / \rightarrow[\mathrm{e}], / \mathrm{y} / \rightarrow[\phi]$, et $/ \mathrm{u} / \rightarrow[\mathrm{o}])$ existe également; eune 'une', asseuré 
Cette tendance à fermer les voyelles moyennes demande une explication. Nous nous rangeons avec H. Vogt (1954) qui souligne qu'un système linguistique n'est capable de subir qu'un nombre limité d'innovations à un moment donné dans le temps, et que le système linguistique lui-même conditionne ceux qui peuvent en dériver. L'interférence linguistique affectera un système dans la mesure où les éléments étrangers correspondent aux possibilités d'innovation inhérentes au système récipient.

Or, pour le cas du métis, nous savons que la tendance à fermer les voyelles moyennes existe à différents degrés dans de nombreux dialectes français. Poirier (1975) a noté qu'en ancien québécois $o$ inaccentué se fermait souvent en ou (clousture 'clôture', cousteau 'côteau', pouchette 'pochette', etc.) et que $e$ inaccentué se fermait en $i$ devant une autre voyelle (agriable 'agréable', criature 'créature', giant 'géant', Montrial 'Montréal', etc.) ou devant une consonne palatale (milleur 'meilleur'). Ces tendances étaient déjà attestées en ancien français et sont demeurées vivaces dans presque tous les dialectes d'oïl. On sait aussi qu'en acadien, /o/ se prononce souvent [U] devant une nasale: [pUm] 'pomme', [estUma] 'estomac'; $/ \phi$ / se prononce souvent [y]: [yzem] 'Eugène', [yrop] 'Europe', [ykaristi] 'eucharistie'. En cajun, un mot comme 'rôti' se prononce [ruti].

En cris, le système vocalique est le suivant (avec les valeurs phonétiques approximatives):

$\bar{i}[\mathrm{i}]$

$\begin{array}{lcc}\mathrm{i}[\mathrm{I}] & & \mathrm{o}[\mathrm{U}] \\ \mathrm{e}[\mathrm{e}] & \overline{\mathrm{o}}[\mathrm{o}] & \\ \overline{\mathrm{a}}[\mathrm{a}] & \mathrm{a}[\Lambda] & \end{array}$

On notera premièrement la présence des voyelles brèves [[]] et [U]. Par contre, il existe une assez grande instabilité dans la réalisation phonétique de ces phonèmes. Selon Douaud (1980), le dialecte des Plaines parlé dans le nord prononce le $\bar{e}$ en [i] (pēyak 'un' [pijsk]) alors que le dialecte des Plaines tel qu'il se parle dans le sud le prononce en [e] ([pejsk]). À Whitefish Lake, en Saskatchewan, $\bar{e}$ et $\bar{l}$ ont été interchangés dans certains mots: nistī [nIstis] 'mon frère aîné' mais nisēmis [nIsemIs] 'mon frère cadet' alors qu'au sud de la province on aura plutôt nistés [nıstes] et nisimis

'assuré', tol 'tout', trové 'trouvé', por 'pour', vos 'vous', covercle 'couvercle', etc. Malheureusement, les seules indications de ce phénomène nous proviennent de textes écrits et il nous faudrait donc des données orales plus fiables pour pouvoir le confirmer. 
[nIsimls], Dans la région du Lac-la-Biche, Alberta, $\dot{e}$ et $\dot{o}$ se prononce toujours [I] et [U] ${ }^{13}$.

On notera également que $\bar{e}$ et $\bar{o}$ (lorsque prononce [e :] et [o: :]) sont phonétiquement plus longs que les [e] et [o] équivalents en français et qu'en fait de longueur, ceux-ci ressemblent plus aux [I] et [U] du cris.

$\mathrm{Vu}$ la variabilité des réalisations phonétiques des voyelles fermées et moyennes du cris, vu la distinction phonologique et phonétique entre voyelles longues et brèves qui font que les brèves $[\mathrm{I}]$ et $[\mathrm{U}]$ du cris s'apparentent auditivement aux [e] et [o] du français, et vu la tendance historique interne au français de fermer les moyennes, il est permis de croire que cette tendance a été renforcée par une situation plus ou moins correspondante en cris pour donner lieu à une innovation en métis.

Mentionnons quelques autres particularités vocaliques du métis. Comme dans certains parlers acadiens, $/ \varepsilon /$ se prononce la plupart du temps [æ] devant $/ r /:$ [pær] 'père'. Souvent, la voyelle fermée antérieure labialisée se produit sans labialisation. On entendra donc [li] pour 'lu' et [vl] pour 'vu', etc. Aussi, toute voyelle en contact avec une consonne nasale a tendance à être nasalisée. Ce phénomène est fort commun en cajun ainsi que dans les créoles français tant des Antilles que de l'océan Indien: [kũmã] 'com-

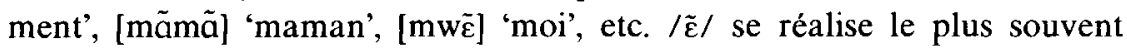

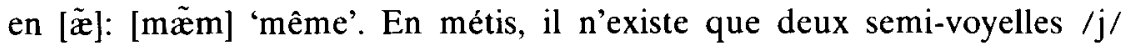
et $/ w /, ; / y /$ est soit effacé comme dans [pll] 'pluie', soit réalisé [w]. Comme dans certains dialectes du Québec, /wa/ se prononce [we] comme dans [awer] 'avoir', [nwer] 'noir', etc.

5.2 Le système consonantique du métis ne diffère sensiblement de celui des autres parlers français que par une règle d'assibilation des dentales qui se réalisent, donc $[t]$ et $\left[\mathrm{d}_{3}\right]$ devant les voyelles antérieures fermées et les semi-voyelles, et non [ts] et [dz] comme en québécois. On entendra

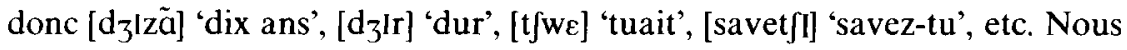
avons déjà noté que la fermeture du /e/ en [I] déclenche systématiquement l'assibilation palatale de la dentale précédente, ce qui démontre bien la systématicité du phénomène: [parãtfl] 'parenté', [kUtfi] 'côté', [klUtfIr] 'clôture'.

13. Douaud 1980 , p. 402 
Ici aussi, il est loisible d'invoquer une influence cris. Le système des consonnes cris contient les dix consonnes suivantes :

$\begin{array}{llll}\mathrm{p} & \mathrm{t} & \mathrm{c} & \mathrm{k} \\ & \mathrm{s} & & \mathrm{h}\end{array}$

m n

j w

L'opposition sourd-sonore n'est pas distinctive en cris, mais normalement on n'aura qu'une réalisation phonétique voisée à l'intervocalique. /c/ se prononce variablement [ts] ou [t $\mathrm{f}]$ et $/ \mathrm{s} /[\mathrm{s}]$ ou [f] (ou $[\mathrm{dz}],\left[\mathrm{d}_{3}\right]$ et $[\mathrm{z}],[3]$ à l'intervocalique). Plusieurs auteurs ont par ailleurs souligné que les variétés chuintantes sont plus communes dans les parlers occidentaux du cris, surtout devant la voyelle /i/. On peut donc facilement concevoir que chez les Métis, une tendance dialectale bien connue du français, celle d'assibiler les dentales devant les voyelles antérieures fermées, a été renforcée par l'existence d'un phénomène semblable en cris. De plus, la tendance du cris à palataliser cette affriquée, surtout devant /i/, aurait été suffisante pour influencer de façon marquée les habitudes articulatoires du métis et d'ainsi systématiser cette variante palatale. Notons finalement que le métis conserve un [h] dans certaines expressions (emphatiques, surtout), tout comme dans certains parlers québécois: [zānehỹm] 'J'en ai une!' Par contre, nous n'avons pas noter la réalisation en $[\mathrm{h}] \mathrm{de} / 3 / \mathrm{comme}$ il se fait encore entendre dans certaines régions du Québec.

5.3 Un des traits les plus caractéristiques du métis est l'emploi de la consonne [1] dans des expressions comme les suivantes:

(1) a. Ça [1] a de l'argent.

b. On [1] avait été sauter.

c. J'ai jamais vu une personne qui [1] est bête...

d. I [1] ont venu pour nous vendre un chevreuil.

e. Je [l] a décidé partir...

Ce phénomène mérite une analyse un peu plus détaillée. Notons d'abord que la présence de ce [1] n'est pas un phénomène unique au métis, puisqu'il est également bien connu en québécois. Dans son analyse de ce [1] non étymologique, Morin (s.d.) démontre premièrement que ce [1] ne peut être analysé comme étant simplement un pronom de reprise car en (la), le pronom de reprise aurait été en; il ne saurait être pronom de reprise en (lb) 
puisque sauter est l'équivalent du verbe 'danser' en français et est donc intransitif. De toute façon, lorsque le pronom clitique le est effectivement présent, nous obtenons une consonne géminée, comme dans ces exemples :

(2) a. S'il fallait acheter une bouteille pour les vieux, et bien, on [11] achetait!

b. Il [j] a parti pis i [II] ont jamais revu.

c. L'église, je crois qu'i [ll] ont vendue au gouvernement...

Morin (op. cit.) établit également une distinction formelle entre le [1] employé avec le clitique sujet ça, comme dans (la) et le [1] employé avec d'autres sujets. En québécois, comme en métis d'ailleurs, le [1] employé avec ça peut être suivi de n'importe quel verbe à initiale vocalique. Nous avons relevé, entre autres :

(3) a. La veille, ça [l] arrivait à l'entour...

b. Charles, ça [1] appelait, celui-là...

c. Ça [l] enveloppait..,

Lorsque [l] suit d'autres pronoms clitiques sujet ou même des formes nominales, seule une forme du verbe avoir ou être peut suivre, comme dans $(\mathrm{lb}-\mathrm{d})^{14}$. C'est pour cette raison que Morin (op. cit.) considère le [1] employé avec ça comme une vraie 'consonne de liaison' (au même titre que le [z] dans une expression française telle que Ils [z] ont mangé, alors que le [1] employé avec d'autres sujets pronominaux ou nominaux serait simplement un [1] agglutiné au verbe avoir ou être puisque la liaison après un syntagme nominal est totalement disparue en français moderne. Les exemples suivants en métis militent effectivement en faveur d'une analyse de [1] comme consonne agglutinée au verbe.

Premièrement, les exemples en (4) démontrent que [1] peut être présent après une variété de syntagmes nominaux à finale vocalique :

(4) a. Pis mon garçon [1] a été en guerre...

b. Quand les mots-là [l] ont parti...

c. La pluie [1] a bien pris...

d. Le petit gars [l] a parti, là...

e. André [l] a eu ses vacances...

14. Morin (s.d.) cite des exemples de [1] avec les seuls clitiques sujet on et vous. En métis, [1] semble pouvoir être employé avec toutes les formes pronominales sujet comme le démontrent les exemples en (1). 
Aussi, nous avons relevé de nombreux exemples de [1] là où une liaison serait carrément impossible puisque le syntagme nominal se termine par une consonne :

(5) a. Mon père [l] a arrivé...

b. Le cour [1] a arrêté...

c. Je pense que l'église [l] a été bâtie...

d. Les épinettes [l] ont une autre couleur, là.

e. Pis, l'autre [1] était parti avec un fusil.

Finalement, il existe une règle en métis qui permet d'effacer le pronom sujet clitique, surtout lorsque l'identité du référent a été préalablement bien établie, comme le montrent les exemples suivants :

(6) a. Paraît que c'était les meilleures femmes qu'il y avait pas là! Paraît que ça dit rien, ça! __ se contentait avec rien! __ se plaint jamais!

b. Un bon jour, il s'en vient ici, pis il me dit... __ commence à parler, __ pas dit grand-chose...

(Nous discuterons davantage ce phénomène en 6.1)

Or, en métis, [1] peut apparaître avec une forme finie du verbe avoir ou être même lorsque le pronom sujet a été effacé, comme dans les exemples suivants :

(7) a. Ils ont été à la chasse; __ [1] ont mis leur tente dans le bois, comme de raison. Là, [l] ont parti à la chasse, là... à pied, là.

b. La pluie [l] a bien pris. Oui, [1] a pris fort.

c. C'était un nommé B...; [l] avait une grosse autruche, pis venait à l'aval ici, à Batoche...

d. Pis Papa, lui, et bien [1] était mort...

e. L'autre [l] a son chez-eux. Tous les trois [I] ont leur chez-eux. Oui, oui, [1] ont tous leur chez-eux.

f. La maîtresse d'école-là, [l] a toutes de ses mémoires de toutes ces vieilles histoires par des livres.

Il ne peut donc s'agir ici de liaison puisque le sujet qui pourrait déclencher la liaison a été effacé. Force nous est alors d'analyser ce [1] comme une consonne qui s'est agglutinée à une forme finie du verbe avoir ou être. Notons, par ailleurs, que ce phénomène est fort complexe puisque ce [1] ne s'agglutine pas de façon absolue. Nous avons noté de nombreux 
exemples où il n'apparaît pas, même dans des contextes où il devrait normalement le faire ;

(8) a. Ça a changé ça, astheure.

b. Ça allait chercher ça à Prince-Albert.

c. Quand ça a arrêté à Batoche...

Des fois, dans une même suite de phrases, le même clitique peut être accompagné ou non du [1] :

d. Il avait envie d'aller dans le B.C.-là. Bien, on [n] a pris mon char pis lui a mené, pis on [1] a été parti pour deux semaines...

e. Ça [l] a de l'argent mais ça a pas de bon sens!

f. On pense qu'on [1] est fin... On se pense toujours plus fin qu'on [n] est !

Les données que nous possédons ne nous permettent pas pour l'instant de déterminer les facteurs qui pourraient jouer dans l'application variable de cette règle d'insertion ou d'agglutination.

On notera finalement que lorsque en précède un verbe dont le sujet est $c ̧ a$, nous obtenons le plus souvent une liaison en [n], tout comme en québécois :

(9) a. Ça [n] en parlait...

b. Ça $[n]$ en voulait...

Néanmoins nous avons relevé au moins un exemple où [1] est employé avec en :

c. C'est ça que ça [l] en font...

6. Quelques aspects du système morphologique du métis.

6.1 Le métis se distingue également des autres parlers français d'Amérique par certains aspects de sa grammaire. Dans ce parler, bien que les distinctions de genre (masculin et féminin) existent, elles sont souvent abolies pour les pronoms personnels de la troisième personne du singulier et du pluriel ainsi que pour les démonstratifs, alors qu'en québécois, par exemple, la neutralisation du genre n'existe qu'à la troisième personne du pluriel du pronom personnel ${ }^{15}$. Ainsi, on aura :

15. Selon Douaud (1980), cette même neutralisation du genre s'applique lorsqu'un Métis francophone parle anglais. 'My grandmother, when he died, he was a hundred and five.' 'She's a good runner, him!' 
(10) a. Ton garçon, elle est smatte...

b. La mousse, il se fait où ce qu'i [1] est à l'abri du soleil.

c. Les petites filles-là... il y a ceux-là et pis celui-là...

Nous savons que le cris distingue lui aussi deux genres mais alors que le français établit une distinction entre le masculin et le féminin, le cris l'établit sur une base de l'animé et du non-animé. Il se peut donc que la neutralisation partielle du genre en métis soit due à une certaine interférence de la part du système classificatoire du cris.

D'autre part, si la distinction du genre s'est maintenue dans tous les parlers français du continent, elle tend à être réduite à certains adjectifs dans plusieurs dialectes et elle est fortement menacée dans le système des déterminants. À Frenchville, par exemple, le démonstratif se réduit à [st] devant une voyelle et à [stə] devant une consonne, quel que soit le genre du déterminé ${ }^{16}$. En cajun, certains adjectifs, variables en français standard, sont invariables : sec, chaud, etc. Même en français populaire, on retrouve Elle était furieux, ma boisson sec, etc ${ }^{17}$.

Évidemment, l'emploi du clitique ça, tant pour référer à un antécédent singulier que pluriel, permet au locuteur métis de se soustraire au problème du genre. Nous croyons d'ailleurs que ceci explique son emploi extrêmement fréquent. Il est à remarquer à cet égard que lorsque ça renvoie à un pluriel, le verbe s'accordera lui aussi au pluriel :

(11) a. Mes neveux, ça [l] ont parti...

b. Eux-autres itou, ça [l] ont fait une revue...

c. I' sont plus que nous-autres... C'est ça que ça [1] en font.

d. Les gens de Winnipeg, quant ça viennent ici...

e. Icitte, ça va bien. Tout le monde, ça sont pas pire... ${ }^{18}$.

6.2 Nous avons vu également aux exemples (6) et (7) qu'en métis, le pronom sujet peut être effacé, ce qui permet encore une fois au locuteur métis de se soustraire au problème de sélection pronominale en fonction du genre du référent. Ici encore, l'influence de la langue cris peut être responsable. En cris, il n'existe de pronoms personnels qu'à la première et à la deuxième personne, la troisième personne étant marquée uniquement par un suffixe verbal :

16. Caujolle (1972), cité dans Valdman (1980).

17. Bauche (1946), pp. 85 et 86 .

18. Les exemples (11a et 11e) sont cités dans Lussier, A et B. Sealey (eds) (1980). 
(12) a. ni pimipahtān

'je cours'

b. ki pimipahtān

'tu cours'

c. pimipahtāw

'il court'

Il se peut donc que l'absence (superficielle) de pronom à la troisième personne en cris soit à la source d'une construction semblable en métis. De l'effacement du pronom à la troisième personne, le métis l'a généralisé à toutes les personnes. Même si dans notre corpus, la très grande majorité des cas d'effacement le sont pour la troisième personne, nous avons relevé ces quelques exemples :

(13) a. Je pense me rappelle cette chanson-là; je pense peux la chanter.

b. Oui, oui, vivait pas riche, savez-tu, mais on était heureux!

6.3 On aura remarqué que le système verbal du métis est réduit et simplifié par rapport au français standard. Au présent de l'indicatif, on obtient les formes suivantes tout comme en québécois :

\begin{tabular}{|c|c|}
\hline $3 \partial \sim 31$ fãt & 'je chante' \\
\hline $\mathrm{t} \int \mathrm{I} \quad \int \tilde{a} \mathrm{t}$ & 'tu chantes' \\
\hline $\mathrm{i} \sim \mathrm{a} \sim \int \mathrm{a} \int a \bar{t}$ & 'il, elle chante' \\
\hline $\int a ̃ t$ & 'nous chantons' \\
\hline fãte & 'vous chantez' \\
\hline $\mathrm{i} \sim \mathrm{a} \sim \int \mathrm{a} \int \mathrm{a} \mathrm{t}$ & 'ils, elles chantent' \\
\hline
\end{tabular}

La seule désinence est donc à la deuxième personne du pluriel. Par contre, les verbes à deux thèmes (verbes en -ir et en -re) ont une forme spéciale à la troisième personne du pluriel : [finIs], [vãd], etc.

La conjugaison du verbe avoir, tant lorsqu'il sert de verbe «réel» que lorsqu'il est auxiliaire, est également réduite :

$$
\begin{aligned}
& 3^{\mathrm{e}} \sim 3^{\mathrm{la}} \\
& \text { ta } \sim \text { tfla } \\
& \text { ja } \sim \text { i } \sim \text { a } \sim \text { jala } \\
& \text { jna } \sim \text { วิla } \\
& \text { vuzave } \sim \text { vulave } \\
& \mathrm{j} \tilde{\boldsymbol{s}} \sim \mathrm{iz} \tilde{\mathrm{s}} \sim \mathrm{i} \sim \int \mathrm{al} \tilde{\jmath}
\end{aligned}
$$

Ce système est presque identique à celui employé à Frenchville ${ }^{19}$ :

19. Ce dernier exemple est cité dans Lussier, A et B. Sealy (eds) (1980). 
(16)

$$
\begin{aligned}
& \text { ze } \\
& \text { ta } \\
& \text { ja } \sim \text { ila } \\
& \text { ôla } \\
& \text { vula } \\
& \text { jõ iló }
\end{aligned}
$$

À l'imparfait, le métis connaît les mêmes formes qu'en québécois, mais à la troisième personne du pluriel, la seule forme connue pour avoir est onvaient et pour être sontaient. Ces formes se retrouvent également pour l'auxiliaire du plus-que-parfait

(17) a. C'est vrai qu'i [1] onvaient bonne tête, ces gens-là...

b. Ces Métis-là... sontaient heureux!

c. I' sontaient bien obligés de freiter...

d. Après qu'ils se sontaient mariés...

e. I [I] onvaient gagné le rébellion...

f. Pis i' sontaient qui [l] onvaient été négligés...

Comme dans de nombreux parlers vernaculaires du français, le métis remplace de façon sporadique le subjonctif par l'indicatif :

(18) a. Avant qu'il va mouiller...

b. Il faut qu'ils faisaient leur ouvrage...

c. Il faut bien que je le fais...

d. Je lui avais écrit qu'il s'en vient...

Aux temps composés, le métis réduit presque toujours l'auxiliaire à une forme de avoir:

(19) a. J'ai venu à St-Laurent.

b. Mon père [l] a arrivé...

c. J'ai resté avec mon p'tit gars.

d. Le cour [1] a arrêté (s'est arrêté)

e. Quand j'ai sorti de l'école...

f. Et pis quand qu'i [l] a venu de l'eau, ces arbres-là, i [1] ont tombé...

g. I' s'a bâti une maison.

h. I' s'onvaient mariés...

Nous avons également noté un certain nombre de "régularisations» de formes verbales, comme dans les exemples suivants :

(20) a. Quoi c'est qu'on apprendait, on comprenait.

b. Ça vaulait pas cher. 
c. Quand même qu'on connaissera l'anglais...

d. I' faudrait que je me marisse...

e. Quand ça voulait pas qu'on save quoi ça disait...

Le clitique en des expressions verbales s'en venir, s'en revenir est réanalysé en métis et fait partie intégrale du verbe, comme le montrent les exemples suivants :

(21) a. I' sont enrevenus...

b. Pis i' sont enrevenus de même, par terre...

c. Je me suis envenue seule...

6.4 Notons, en résumé, les détails grammaticaux du métis suivants que l'on retrouve également, de façon plus ou moins identique, dans les divers parlers français d'Amérique :

1) la confusion entre les pronoms clitiques objet direct et indirect :

(22) a. Personne pour les dire qu'i' sont menteurs.

b. Les autres lui aidaient...

c. Ça les prenaient un mois...

2) l'emploi de la particule interrogative - $t i$ (prononcée [t $\mathrm{t} 1]$ ) comme en québécois. Il est intéressant à noter, à cet égard, qu'en cris il existe aussi une particule interrogative $-c i([\mathrm{ts}] \sim \mathrm{t} f \mathrm{l}])$ qui s'ajoute après la première forme pleine d'une phrase. Leur ressemblance phonétique et syntaxique a sans doute renforcé l'emploi de la particule interrogative - $t i$ en français. On aura remarqué qu'à l'exemple (13b), le métis emploie cette particule dans un contexte où l'interrogative est improbable et où, de toute façon, le sujet est effacé. Il semble plutôt que savez-tu est l'équivalent du québécois $t$ 'sais.

3) l'emploi du conditionnel dans les deux propositions d'une phrase conditionnelle est typique :

(23) S'i [1] auraient marché comme ça, la charrette aurait calé!

4) certains verbes, expressions verbales et expressions adjectivales qui en français standard exigent une préposition, se voient en métis, soit sans préposition soit avec une préposition différente de celle exigée en français standard :

(24) a. Je suis assez fin de savoir que je suis bête!

b. - qui sontaient intéressés de ramasser ces vieilleries.

c. Il a continué parler en cris.

d. Les autres [l] ont commencé aller par dedans le nord. 
e. Quand qu'ils se sont tous décidés partir pour s'en venir icitte...

f. J'avais acheté le morceau de terre en haut l'église.

g. Elle voulait s'en venir rester proche nous autres.

7 Quelques aspects syntaxiques du métis.

7.1 La structure des expressions possessives du métis est particulière à ce parler et semble être directement calquée sur le cris. Dans cette langue, le déterminant possessif est marqué par un préfixe nominal. Ainsi :

$\begin{array}{cl}\text { ni-maskisin } & \text { 'mon soulier' } \\ \text { ki-maskisin } & \text { 'ton soulier' } \\ \text { o-maskisin } & \text { 'son soulier' }\end{array}$

Pour exprimer un lien de possession entre deux substantifs (le soulier de Jean), le cris antépose toujours le "possesseur» à l'objet possédé et celui-ci est toujours marqué par le préfixe possessif de la troisième personne :

(26) Cãn o-maskisin 'lessoulier de Jean'

Jean son-soulier

C'est précisément la structure qu'emploie le métis pour signifier la possession :

(27) a. C'était Maman ses cousins. (C'était les cousins de Maman.)

b. C'est Marie-Jeanne sa fille quand qu'elle s'est mariée.

(C'est la fille de Marie-Jeanne...)

c. C'est un de mon garçon son petit qui était mort.

(C'est le petit d'un de mes garçons...)

d. En allant dans le docteur son petit bois...

(Dans le petit bois du docteur) ${ }^{19}$

Cette construction est très ancienne car le premier à décrire quelque peu le parler métis, A. Elliott (1886), avait noté Pierre son livre pour le livre de Pierre.

L'emploi de cette construction n'est néanmoins pas absolue car nous avons également relevé les expressions suivantes :

(28) a. Ça doit être la mère d'Édouard P...

b. C'étaient les enfants d'eux autres...

c. La fille de mon frère... 
d. Ça se trouve la fille à Alfred, ça.

e. Ça se trouve le grand-père à la femme de mon garçon ${ }^{20}$.

En cris, il existe une classe restreinte de noms (appelés «noms dépendants») qui exigent toujours le préfixe possessif. Ces noms incluent les parties du corps, les expressions de parenté et divers objets considérés comme des possessions importantes (cheval, chien, maison, etc.) Cette particularité du cris est sûrement responsable des expressions suivantes en métis :

(29) a. C'est un de mon oncle qui est avec un de son ami.

b. Papa avec un de sa tante...

c. L'autre mon frère qui est là...

d. Il est marié avec un de ma cousine.

e. Il y en a un autre son neveu qui restait pas loin.

f. Pis ça ici, c'est une autre ma nièce, ça.

g. ...Le vieux Charles $N$.... son grand-père à ma femme...(le grandpère de ma femme)

L'exemple suivant démontre le cumul des deux constructions :

h. C'était ma mère son frère. (C'était le frère de ma mère)

Le québécois connaît des expressions comme mon mon-oncle, une matate, une ma-soeur (une religieuse), etc. Par contre, nos données ne nous permettent pas d'affirmer que l'agglutination du possessif s'étend aux termes pour désigner les parties du corps ou à des noms de «possession importante» comme en cris.

7.2 Nous avons discuté en 5.3 le phénomène du [1] agglutiné. Cette habitude a eu des répercussions dans la construction des phrases relatives, surtout lorsque le pronom relatif est sujet. Dans des phrases comme :

(30) a. J'ai jamais vu une personne qui [1] est bête.

b. C'était le plus vieux de mon frère qui [l] était marié.

c. Mon garçon, celui-là qui [l] était dans l'armée...

nous pouvons analyser [1] comme une consonne d'agglutination, ce qui maintient la forme normale du pronom relatif. Par contre, ces phrases pourraient être analysées de la manière suivante :

20. On notera ici l'absence de la copule. Nous avons relevé onze exemples de l'expression Ça se trouve... dans notre corpus et neuf fois sur onze, le verbe être était absent. Aucune autre instance d'effacement du verbe être copule n'a été remarquée. 
(31) a. J'ai jamais vu une personne qu'i [1] est bête.

où le pronom relatif qui serait réanalysé en simple complémenteur qu(e) et où la fonction sujet serait remplie par le pronom personnel $i(l)$. Nous croyons que c'est précisément cette réanalyse qu'effectue le métis, du moins lorsque l'antécédent du relatif est féminin, puisque nous avons relevé de nombreux exemples :

(32) a. C'est un de ma fille qu'elle est mariée. [kalemarje]

b. C'est ma fille qu'elle avait peur. [kalavepor]

c. C'était Maman qu'elle lisait. [kallize]

d. C'est Maman qu'elle restait ici. [kalreste]

Dans les exemples (32a et b) nous ne pouvons savoir si le [1] appartient proprement au pronom ou s'il fait plutôt partie du verbe avoir ou être à titre de consonne agglutinée. Mais en (32c), étant donné la consonne géminée, force nous est de conclure que [1] fait partie du sujet, ce qui est confirmé par l'exemple (32d)

Tout comme les autres parlers français d'Amérique, le métis semble ignorer la construction relative objet de préposition du français standard. À sa place, le métis permet une proposition «échouée», si celle-ci est une préposition dite «forte» (avec, pour), soit remplaçant une préposition dite «faible» par l'adverbe correspondant (dans $\rightarrow$ dedans, sur $\rightarrow$ dessus), soit encore effaçant une proposition «atone» ( $a$ ou $d e$ ), comme dans les exemples suivants :

(33) a. Ils prenaient la graisse qu'ils voyageaient avec-là...

b. ...les portraits que vous mettez dedans, là...

c. C'est Maman avec ses poules qu'elle donne à manger.

Le métis rẹmplace le plus souvent une construction relative du type ce que... par quoi c'est que... ${ }^{21}$ celle-ci étant souvent réduite phonétiquement à [kwask(a)]:

(34) a. Voyez-tu quoi c'est qu'il fait avec son chien? [kwasck]

b. Je sais pas quoi c'est qu'il était. [kwask]

c. Le mot dit quoi c'est qu'il sert. [kwask]

Par contre, nous avons noté :

d. Vous connaissez quoi je veux dire?

21) Cette construction ne semble pas affecter ce qui. Néanmoins, nous avons noté l'expression : Je répète pour me souvenir quoi qui s'en vient. 
e. Je me contentais avec quoi j'avais.

f. On faisait qu'est-ce qu'on pouvait.

La forme pleine ou réduite de c'est que se retrouve également dans de nombreuses formes interrogatives (directes ou indirectes) :

(35) a. Pourquoi c'est que Dieu m'a conservé? [søk]

b. ...où c'est que la boucane qu'i' passe, là... [sck]

c. I' s'en va où c'est qu'il y avait des vieilles... [sk]

d. Pourquoi c'est que tu prends cette sauvagesse-là? '[skə]

e. Comment c'est qu'i 1 a attrapé...? [sk]

f. Quoi c'est que c'est ça, l'argent? [skə]

Nous avons également obtenu :

g. Quoi je vais en faire de cet argent-là?

h. Quoi je dirais bien?

i. Quoi c'est ça?

7.3 Finalement, en métis, on ajoute souvent un que "conjonctif» après certaines locutions adverbiales :

(36) a. Quand qu'il veut venter...

b. Quand qu'ils sontaient tannés de me voir pleurer...

c. Je sais pas combien qu'ils ont dit d'animaux qu'ils onvaient.

d. Quand même que t'es après travailler...

e. Mon père parlait un peu français mais pas le bon français comme qu'i [l] est écrit.

f. On pouvait pas sortir comme qu'on voulait quand que j'étais petit. Lorsque le sujet est un substantif, nous remarquons une désarticulation de l'expression adverbiale conjonctive :

(37) a. Depuis mon mari qu'il est mort...

b. Après le buffalo qu'il est parti...

c. Au B.C. où sa sœur qu'elle reste...

Dans les phrases quelque peu complexes, nous avons remarqué aussi un emploi abusif de complémenteur (ou relatif) que :

(38) a. I' y a bien des histoires que j'aurais appris que si j'avais connu que j'aurais vécu si longtemps que ça.

b. Je sais pas si je mérite que le Bon Dieu qu'il me garde.

c. Je vais peut-être dire que le Bon Dieu qu'il me prenne. 
d. C'est un album que quand je [I] a été au B.C. ma fille qu'elle m'a donné.

e. Où c'est qu'astheure $q u$ 'ils mettent des clous-là, c'était des jouilles de bois qu'ils mettaient.

8. Quelques aspects du lexique du métis.

Le lexique du métis se caractérise par quatre traits principaux : termes (ou expressions) d'origine française typiques du parler français au Canada (surtout québécois), termes d'origine française qui sont soit uniques au métis, soit que malgré leur présence, connus ailleurs, leur sens ou leur acceptation est propre au métis, termes empruntés à l'anglais, ceux-ci étant connus partout au Canada ou uniquement par des locuteurs francophones à l’ouest du Québec, et termes empruntés au cris.

8.1 La plus grande partie du lexique métis se classe dans la première catégorie, c'est-à-dire que la plupart des mots et des expressions employés en métis sont également employés en français du Canada (sinon d'Amérique). À titre d'exemples, mentionnons les termes suivants :

$\begin{array}{ll}\text { être en brosse } & \text { 'soûlade' } \\ \text { gratteux } & \text { 'avare, pingre' } \\ \text { astiner } & \text { 'contredire' } \\ \text { astheure } & \text { 'maintenant' } \\ \text { boucane } & \text { 'fumée } \\ \text { escousse } & \text { 'un certain temps' } \\ \text { jongler } & \text { 'penser, réfléchir' } \\ \text { malle } & \text { 'courrier' } \\ \text { tanné } & \text { 'fatigué, embêté' } \\ \text { caille } & \text { 'peau tachetée, pie' } \\ \text { écarté } & \text { 'perdu' } \\ \text { prendre vent } & \text { 'se remettre à respirer' } \\ \text { menterie } & \text { 'mensonge' } \\ \text { mausus } & \text { euphémisme pour 'maudit' } \\ \text { redressir } & \text { 'redresser' } \\ \text { faire brun } & \text { 'crépuscule' }\end{array}$

Parmi ces termes, on en trouve plusieurs qui, au Québec du moins, sont maintenant vieillis, voire même inconnus de la plupart des locuteurs même s'ils se trouvent dans l'un ou l'autre des lexiques publiés: 


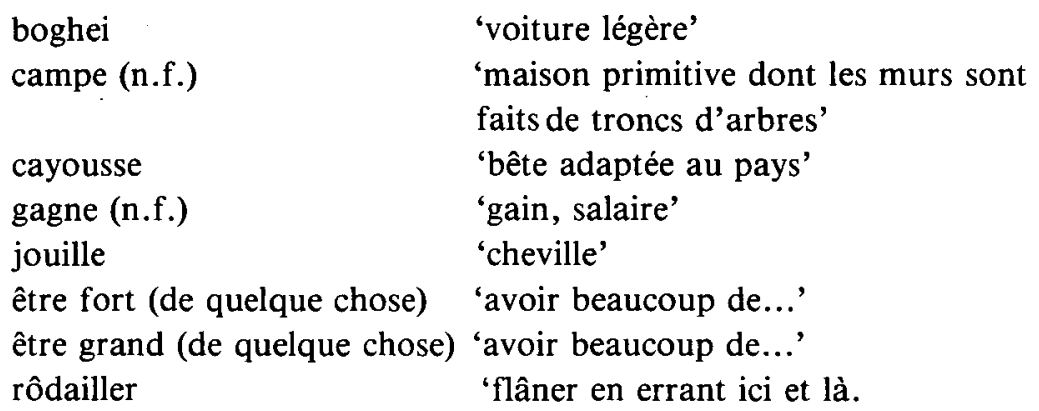

8.2 Un certain nombre de termes semblent être uniques au métis, du moins nous ne les avons repérés dans aucun des lexiques ou glossaires publiés. Parmi ceux-ci mentionnons :

$\begin{array}{ll}\text { bracailler } & \begin{array}{l}\text { 'branconner' } \\ \text { empresser }\end{array} \\ \text { 'marquer le bétail avec un fer pour en } \\ \text { nouque } & \text { 'ndiquer le propriétaire } \\ \text { embête } & \text { 'embêtements' } \\ \text { porte-épic } & \text { 'porc-épic' }\end{array}$

Certains autres termes existent bien en québécois mais le sens qu'on leur donne en métis n'est pas celui qu'on leur donne au Québec :

Québec : 1) papier buvard; 2) marque
gravée à l'aide d'un marteau sur les
billes de bois afin d'en indiquer la
propriété.
métis : fer (chauffé au rouge) qui sert à
marquer le bétail pour indiquer le
propriétaire.
Québec : En plus des sens bien connus
comme 'frontière entre le Canada et les
États-Unis, 'éventail de services ou
marchandises à offrir, 'compagnie de
transport naval ou aérien', 'chemin de
fer' etc., le terme désigne aussi 'chemin
public entre deux cantons ou deux
seigneuries'.
métis : chemin de comté


traille

faire hommage

sauter

cassette

grade (n.f.)
Québec: 'pont volant'

métis : 'chemin de traille' indique une piste pour charettes dans la prairie.

Québec: 'faire une offrande'

métis : 'rendre ses hommages à quelqu'un (dire bonjour)

Québec: (n.m.) degré universitaire. métis : année scolaire (e.g. J'ai pris la grade deux)

Québec : 1) s'élever de terre avec effort et

2) franchir des rapides en canot' métis : 'danser'

Québec : 'valise d'un marchand ambulant, portée sur le dos.' métis : 'sac à dos d'un chasseur'

8.3 Comme on pourrait s'y attendre, le métis abonde en termes empruntés à la langue anglaise. Nombre de ceux-ci sont bien connus en québécois, à tel point qu'ils ne sont même plus perçus comme étant des anglicismes. Ainsi :

$\begin{array}{ll}\begin{array}{l}\text { fleur (flour) } \\ \text { smatte (smart) }\end{array} & \begin{array}{l}\text { 'farine' } \\ \text { 'intelligent, bien élevé, } \\ \text { gentil' }\end{array} \\ \begin{array}{l}\text { 'achâdrer (bother) } \\ \text { mouver (move) }\end{array} & \begin{array}{l}\text { 'déménager, changer de } \\ \text { place' }\end{array} \\ \text { ouagine (wagon) } & \begin{array}{l}\text { 'grosse charrette à quatre } \\ \text { roues' } \\ \text { 'magasin' }\end{array} \\ \text { store (store) } & \text { 'mise en conserve' } \\ \text { cannage (can) } & \text { 'attelage' } \\ \text { tîme (team) } & \text { 'endurer' } \\ \text { toffer (tough) } & \text { 'fret' } \\ \text { freite, (freight) } & \text { 'flâner' } \\ \text { bommer (bum) } & \end{array}$

D'autres termes sont toujours perçus comme étant des emprunts à l'anglais et sont également connus au Québec : 


\begin{tabular}{ll}
$\begin{array}{ll}\text { soft drink } \\
\text { spray (v. et n.) }\end{array}$ & $\begin{array}{l}\text { 'boisson gaseuse' } \\
\text { 'jet pulvérisé, asperger, } \\
\text { arroser, vaporiser' } \\
\text { bunch } \\
\text { shack }\end{array}$ \\
gopher & 'cande' \\
bootlegger & 'marme' \\
truck & 'trafiquant en boissons \\
alcooliques' \\
easy & 'camion' \\
magasine & 'aisé, facile' \\
watcher & 'revue' \\
roughment & 'regarder, surveiller' \\
moskeg & 'rudement' \\
\hline
\end{tabular}

D'autres encore sont inconnus au Québec; ont un sens particulier au métis, ou ne sont connus qu'à l'ouest du Québec :

\begin{tabular}{|c|c|}
\hline polisher (polish) & 'polir' \\
\hline pasture & 'pâturage' \\
\hline railleder (ride) & $\begin{array}{l}\text { 'monter à cheval, à } \\
\text { bicyclette, etc.' }\end{array}$ \\
\hline Post Office & 'Bureau de poste' \\
\hline free range & 'pâturage sans clôture' \\
\hline carrier (carry) & 'porter' \\
\hline flat & $\begin{array}{l}\text { 'lot de terre le long d'une } \\
\text { rivière' }\end{array}$ \\
\hline boster (bust) & 'mourir' \\
\hline homestead & $\begin{array}{l}\text { 'terre obtenue gratuitement } \\
\text { du gouvernement' }\end{array}$ \\
\hline cabousse (caboose) & 'petite cabane sur traîneau' \\
\hline brîder (breed) & $\begin{array}{l}\text { 'produire et élever des } \\
\text { animaux' }\end{array}$ \\
\hline
\end{tabular}

8.4 Nous avons relevé assez peu de termes empruntés au cris. Il se peut, par ailleurs, que nos locuteurs en connaissent davantage mais qu'ils n'aient pas eu l'occasion de s'en servir à cause des sujets de conversation choisis. Voici les termes cris que nous avons décelés : 


$\begin{array}{ll}\text { kiskayon } & \begin{array}{l}\text { 'animal ayant une queue } \\ \text { courte' } \\ \text { mishatim }\end{array} \\ \text { nanta } & \text { 'appoval' } \\ \text { tanchi } & \text { 'bonjour' } \\ \text { awapou } & \text { 'nourriture' } \\ \text { kankwa } & \text { 'porc-épic' } \\ \text { kiskapatew } & \text { 'fumée' }\end{array}$

\section{Conclusion}

Nous sommes conscient que ces quelques remarques ne font qu'effleurer la structure linguistique du métis. Il reste énormément à faire et à connaître. Nous avons néanmoins tenté d'isoler quelques traits phonologiques, morphologiques, syntaxiques et lexicaux de ce parler qui nous semblaient les plus intéressants. Nous espérons que les quelques faits décrits dans cet article pourront susciter l'intérêt d'autres linguistes pour que ceux-ci puissent pousser plus avant nos connaissances sur ce parler avant qu'il ne disparaisse à tout jamais.

Robert A. Papen

$U Q A M$ 


\section{Références}

BARBEAU, V. (1963), Le Français du Canada, Québec, édition 1970, Librairie Garneau.

BAUCHE, M. (1946), Le Français populaire, Paris, Payot.

BÉLISLE, L-A. (1979), Dictionnaire nord-américain de la langue française, Montréal, Beauchemin.

CLAPIN, S. (1894), Dictionnaire canadien-français, Montréal, Beauchemin.

CONWELL, M. \& A. JUILLAND (1963), Louisiana French Grammar, La Haye, Mouton.

CRAWFORD, J. (1979), "The Standardization and instrumentalization of creole languages : standardization of orthography in Michif», communication présentée à la Conference on Theoretical Orientation in Creole Studies, St. Thomas, Ilses Vierges.

CRAWFORD, J. (1983a), 'Speaking Michif in four Métis communities, ' in Canadian Journal of Native Studies III, I, pp.47-55.

CRAWFORD, J. (ed.) (1983b), The Mitchif Dictionnary, Turtle Mountain Chippewa Cree, Pemmican Press.

DeTREMAUDAN, A. (1936), Histoire de la nation métisse dans l'Ouest canadien, SaintBoniface, $2 \mathrm{e}$ édition, 1979, Les Éditions du blé.

DIONNE, N.E. (1909), Le Parler populaire des Canadiens français, Québec, Laflamme, Proulx.

DOUAUD, P. (1980), «Métis : a case of triadic linguistic economy», Anthropological Linguistics 22, pp. 392-414.

DULONG, G. (1973), «Histoire du français en Amérique du Nord.» in T. SEBEOK (1973) (ed.), pp. 404-421.

DUNN, O. (1880), Glossaire franco-canadien, Québec, édition 1976, Presses Université Laval.

ELLIOTT, A. (1886), "Contribution to a history of the French language in Canada II : Speech mixture in French Canada, external influences", in American Journal of Philology 7, pp. 141-160.

HADEN, E. (1973), «French dialect geography in North America», in T. SEBEOK (1973) (ed.)

LUSSIER, A. \& B. SEALY (eds.) (1980). The Other Natives : Les Métis, vol. III, Manitoba Métis Federation Free Press.

MORIN, Y-C. (s.d.), «Les [I] non étymologiques après ça et les autes clitiques dans le français de Québec.», manuscrit, Université de Montréal.

PAPEN, R. (1975), "Les dialectes français de l'Amérique du Nord», CASLT Bulletin, 1975.

POIRIER, C. (1975), «La prononciation québécoise ancienne d'après les graphies d'un notaire du XVII ${ }^{e}$ siècle», in JUNEAU, M \& G. STRAKA (eds), Travaux de linguistique québécoise, Québec, Presses de l'Université Laval.

PRÉFONTAINE, R. (1980), «Le parler métis», in LUSSIER, A. \& B. SEALY (eds) (1980)

PUPIER, P. \& R. PELCHAT (1972), «Observations sur la phonologie des pronoms personnels du français montréalais", Lingua 29, pp. 326-346.

RHODES, R. (1976), «French Cree - A case of borrowing.» in W. COWAN (ed.) Actes du $8^{e}$ Congrès des Algonquinistes, Carleton U. d'Ottawa.

ROGERS, D. (1977), Dictionnaire de la langue québécoise rurale, Montréal, VLB Éditeur. 
SEBEOK, T. (ес.) (1973), Current trends in Linguistics, Vol. 10: Linguistics in North America, La Haye, Mouton.

SOCIÉTÉ DU PARLER FRANÇAIS AU CANADA (1930), Glossaire du parler français au Canada, Québec, Presses Université Laval.

VALDMAN, A. (1974), «Le parler vernaculaire des isolats français en Amérique du Nord.» Revue de la Louisiane 3, pp. 43-58.

VINAY, J-P. (1973), "Le français en Amérique du Nord : Problèmes et réalisations», in T. SEBEOK (1973)

VOGT, H. (1954), «Language contacts», WORD 10, pp. 365-374,

WOLFART, H.C. \& J. CARROLL (1973), Meet Cree: A Guide to the Cree language, Edmonton. University of Alberta Press. 